\title{
EN TORNO A LOS VERBOS PERIFRÁSTICOS DEL ESPAÑOL: UN ANÁLISIS SINTÁCTICO TRANSFORMACIONAL $\left(^{*}\right)$
}

o. Contenido del ensayo.

Intentaremos demostrar: prinero, que los verbos perifrásticos del espaniol no son verbos auxiliares desde un punto de vista puramente furmal, y segundo, que las características de estos verbos deben y pueden ser explicadas nediante reglas de transformación y reglas de interpretación seinántica.

Lil cnsayo se divide en tres partes: en el primer párrafo, introduciremos las características de los verbos perifrásticos que parcceil ser la bisse de los estudios dedicados al problema; en el segundo párrafo, demostrarcmos que no hay un solo argumento válido para considerar lus verbos perifrásticos como verbos auxiliares; en el tercer párrafo, se esbozará cómo las características de los verbos perifrásticos pueden y debeu ser explicadas.

\section{Introducción.}

Iis muy curioso el paralelismo sintáctico ${ }^{1}$ que se halla entre oraciones como p. ej.:

(1)a. Yedro aprende el español

b. Pedro ha aprendido el español

- No se trata aqul de lacer una apologia de la gramática generativa transformacional, ni una introducción a ella. Sólo es un estudio sintáctico cuya base teórica doy por conocida.

1 Sintáctica será toda relación que se basa en el modo de combinarse las palabras, morfemas, etc. 
c. Pedro está aprendiendo el español

d. Pedro comienza a aprender el español

e. Pedro ha de aprender el español

En todas estas oraciones, los sintagmas: Pedro, aprender ${ }^{1}$, el español, prescutan las mismas relaciones sintácticas y semánticas. Asi, $P c d r o$ es noubre de persona, sujeto, agente; aprender es verbo de acción; el español es sintagma nominal, objeto paciente; etc. Podrfa decirse que, siendo. (I)a. la oración primitiva; (I)b.' consta de (I)a. más haber, (I)c. de (I)a. más estar, etc. De ahi que haber, estar, comenzar y haber de, hayan sido considerados como verbos auxiliares o verbos que, combinándose con'otro, forman una especie de perifrasis verbal, en la cual aprender es el núcleo y haber, etc. el modificante.

Estos verbos auxiliares o perifrásticos añaden alguna que otra determinación aspectual, modal o temporal a la oración primitiva entera. Parece que los verbos perifrásticos desempeñan el mismo papel que las desinencias verbales $-e,-\{a,-i \delta,-e r a ́,-e r i a$, que representan la forma del Presente, el Imperfecto, el Pretérito, el Futuro y el Condicional, respectivamente, en las oraciones siguientes:

(2)a. Pedro aprende el español.

b. Pedro aprendía el español

c. Pedro aprendió el español ..; :

d. Pedro aprenderá el español :

e. Pedro aprendería el español

Hay más todavia: no se puede cambiar el orden: verbo perifrástico más verbo, como tampoco puede alterarse el orden: verbo más desinencia verbal.

Finalmente, es inconcebible que el verbo perifrástico o la desinencia verbal tengan relaciones directas con el sujeto o el objeto, es decir, . no hay oración en que el sujeto o el objeto prohiba o restrinja el uso de ningún verbo perifrástico ni de ninguna desinencia verbal.

I.I. El paralelismo entre las desinencias verbales y los verbos perifrásticos constituye un argumento en pro de la hipótesis de que, en español, debe distinguirse una clase de verbos auxiliares. Sin embargo; no hay paralelismo total: en primer lugar, cada verbo exige una desi-

1 Mre adhiero a la costumbre de citar los verbos por la forma del infinitivo.. Al seguir esta práctica, no doy a entender que considero el infinitivo más básico que las otras formas verbales. 
neucia verbal; la presencia de un verbo perifrástico, por el contrario, no es obligatoria; en segundo lugar, los mismos verbos perifrásticos exigen desinencias verbales, y, con la única excepción de soler ${ }^{1}$, la conjugación de dichos verbos no es difcrente de la de los verbos noperifrásticos. Listas diferencias que hay entre las desinencies verbales y los verbos perifristicos constituyen argumenitos cn contra de la hipótesis que acabamos de exponer.

r.2. A primera vista, parece que hay un equilibrio entre los argumentos cu pro de la hipótesis arriba expuesta y los argumentos en contra de la misma. Pero se verá que, en una teoría sintáctica formalizada, sólo quedarán en pie estos últimos; resultará que los argumentos en pro carccen de una base sintáctica y que los fenómenos en los que se basan deben ser explicados de otra manera.

2. Los verbos auxiliares en una gramálica transformacional del español.

2.0. IIadlich (r971:59) propone más o menus las siztiduntes reglas de rescritura para el español:

$\mathrm{R}(\mathrm{i}) \quad \mathrm{S}^{2} \longrightarrow \mathrm{NP} \quad \mathrm{AUX} \cdot \mathrm{VP}$

(ii) $\mathrm{AUX} \longrightarrow$ TMP (M-r) (haber-do) (NI-r) (MI-I) (esiar-11du)

(iii) ' $\mathrm{NMP}^{3} \longrightarrow$ Presente, Imperfecto, Prctérito, Futuro, Condicional

(iv) $\mathrm{M} \longrightarrow$ poder, soler, haber de, parecer, deber (de), acabar de, tener que, tralar de, dejar de

Como las desinencias propias del infinitivo, el participio $y$ el gerundio tienen que colocarse detrás del 'verbo' siguicnte, ell cl lugar donde se hallan en la estructura superficial, Hadlich propone una regla de transformación, 'T-afijo:

1 Soler, en muchos dialectos, sólo se usa en el presente $y$ el imperfecto. Es rara la forma del pretérito, el infinitivo, el participio pasado, y sobre todo el gerundio. No se usan las formas del futuro $y$ el condicional.

- Iixplicación de los simbolos: $S=$ oración; $\rightarrow=$ se rescribe como; $N P=$ sintagma nominal; $A U X=$ (elemento) auxiliar; VP $=$ sintagma verbal; TMP $=$ desinencias de tiempo $\mathrm{y}$ aspecto; $\mathrm{M}=$ (¿verbo?) modal; $\mathrm{r}=$ desinencia del infinitivo; do = desinencia del participio pasado; 11 do = desinencia del gerunàio.

- Hewos simplificado un poco la regla de Hadlich. 
$\mathrm{T}$ (i) T-afijo

$\frac{X}{I}-\frac{\text { Af }}{2}-\frac{V}{3}-\frac{Y}{4} \longrightarrow I-3+2-4$

Condicioues: $\quad \Lambda f=T M P, r$, do, ndo

$\mathrm{v}=\mathrm{M}$, haber, estar, $\mathrm{V}$

Sen la oración:

(3) Juan podria haber estado trabajando

cuya estructura subyacente se representa por:

(4) Juan-Condicional - poder - r - haber - do-estar - ndo - trabajar.

Aplicando repetidas veces $T$-afijo, se deriva la estructura superficial:

(5) Juan - poder + Condicional - haber + $\mathrm{r}$-estar + do - trabajar + + ndo.

Ista sirve de entrada ${ }^{1}$ al componente fonológico, el cual derivará de ella la representación fonética ${ }^{2}$ que corresponde a (3).

A continuación estudiarenos las consecuencias del punto de vista de Hadlich.

2.I. Hay fenómenos sintácticos que se explican de una manera adecuada mediante reglas de rescritura; otros fenómenos se captan mejor utilizando reglas de transformación. Al intentar explicar peculiaridades sintácticas de verbos como poder, etc. mediante reglas de rescritura, hay que darse cuenta de las propiedades formales de éstas.

En primer lugar, generando los verbos modales mediante una regla de rescritura y los demás mediante otra, damos a entender que los verbos modales pertenecen a una categoría sintáctica y los demás verbos a otra.

En segundo lugar, el orden de sucesión de los elementos que constituyen una expansión derivada mediante una regla de rescritura debe ser fijo, o, por lo menos, debe haber un orden básico.

En tercer lugar, se utilizan las reglas de rescritura solamente si es posible limitar de una manera natural la totalidad de los elementos

1 'Entrada' es la traducción del inglés 'input'; creo que es término más usual que 'inducto', traducción propuesta por Otero (1970).

2 Por lo que se refiere a la teoria general de este componente, véase Chomsky y Halle (rg68). Harris (1969) es un excelente estudio del componente fonológico del espaũol. 
que se encuentran en las posiciones indicadas. Es decir, que, según Hadlich, la expansión máxima $R(i i)$ será:

$\mathrm{TMP}-\mathrm{M}+\mathrm{r}-$ haber $+\mathrm{do}-\mathrm{M}+\mathrm{r}-\mathrm{M}+\mathrm{r}-$ estar $+\mathrm{ndo}$

lo que corresponde a una oración como por ejemplo:

(6) Juan debe haber tenido que dejar de estar trabajando

excluyéndose toda oración que exceda a estos límites, como por ejemplo:

(7) Juan suele deber de haber tenido que dejar de estar trabajando

Is obvio: r. $^{\circ}$ que no hay una razón a priori para distinguir entre verbos y 'modales', ya que ambos exigen las mismas desinencias; $2 .^{\circ}$ que es difícil establecer un orden de sucesión básico entre los 'modales' que enumera Hadlich; y $3 .^{\circ}$ que es poco menos que imposible limitar de un modo natural el número de 'modales' que pueden combinarse entre ellos.

De alú que el análisis de Hadlich sea inadecuado. Los mismos argumentos en contra de tal análisis ya se hallan en Klein (rg68). Pascmos revista a los detalles de la argumentación.

2.2.0. En obras gramaticales descriptivas del inglés, el alemán y el francés hay discusiones valiosas acerca de los miembros de que consta cl constituyente AUX. Veamos primero los argumentos que han sido utilizados y las conclusiones que se han sacado de los mismos.

2.2.I. Chomsky (1957:39) propone rescribir el elemento AUX de la manera siguiente:

$\mathrm{R}(\mathrm{v}) \mathrm{A} J \mathrm{JX} \longrightarrow \mathrm{C}^{1}(\mathrm{M})$ (have - en) (be - ing) ${ }^{2}$

Si se compara $\mathrm{R}(\mathrm{v})$ con $\mathrm{R}$ (ii), se notará que $\mathrm{C}$, have - en, y be - ing cu $R(v)$ son equivalentes a TMP, haber - do, estar - ndo, respectivamente, en $R$ (ii). Los 'modals' del inglés se definen mediante:

$\mathrm{R}$ (vi) $\mathrm{M} \longrightarrow$ will, can, may, shall, must

1 Explicación de los simbolos: $\mathrm{C}=$ desinencia de número, persona y tiempo; en a desinencia del 'past participle'; ing = desinencia del 'gerund'.

2 Hago caso omiso de (be - en), ya que el mismo Chomsky dice, unas pacas páginas más abajo, que no forma parte de $A U X$. 
Los argumentos que aduce Chomsky para justificar la distinción tajante entre 'modals' (que no son verbos), y los verbos, los cuales sou. miembros de la clase $\mathrm{V}$, son los siguientes.

Primero, el orden de $\mathrm{M}$, have, be, es inalterable:

(8)a. John may have been working

'Juan puede haber estado trabajando'

b. *Jolun has may (?) be working

'Juan ha podido estar trabajando'

c. "Jolnn is having may (?) work

'Juan está habiendo podido trabajar'

Segundo, es imposible colocar un 'modal' después de otro. Esto lo demuestra el ejemplo de Jackendoff (Ig68: (4I)):

(9) *I should can use two modals in a row if they are verbs

'Debía poder usar dos modales contiguos, si son verbos'

'Tercero, hay varias reglas de transformación en inglés cuya descripción estructural debc mencionar AUX, pero no V. Es decir que éstas. se aplicau a los miembros de AUX, a saber, $\mathrm{C}, \mathrm{M}$, have, be, pero no. a los $\mathrm{V}^{3}$. Me limito a indicar las reglas por medio de ejemplos.

Sea un $V$, want 'querer'; $y$ un $M$, will 'ir $a$ ':

(I0)a. John wants to roork

'Junil quicre trabajar'

b. Joln will work

'Juan va a trabajar'

- en oraciones interzogativas, want exige la presencia de do 'hacer' y will no 1.2 aigc, siendo el stijeto pọstpuesto a do, 'si hay want, y a will, si éste cstá preseistc:

(II)a. does Joln want to work? (y. no:.* wants Joln to work?)

'Juan ¿quicre trabajar?'

b. will Join work? (y no: * does John will work?) 'Juan ¿va a trabajar?'

- en oraciones negativas, si solamente want está presente, la negación no debe ser precedida por do; en caso de que en la oración figure will éste debe anteponerse a no, no pudiendo emplearse do:

1 Véause especialmente Clomsky (1957: 6r ss.) y Klima (1964). 
(12)a. John does not want to work (y no: * John wants not to work) 'Juan no quiere trabajar'

b. Joln won't ( $=$ will not) work (y no: *John does not will work) 'Juan no va a trabajar'

- cn las llamadas 'tag questions', se repite AUX, o sea do + $\mathrm{C}$, si solamente reant estál presente; con reill, se repite éste y no se usa do:

(I $\left.2^{\prime}\right)$ a. Joln wants to work, doesn't he? (y no: *...., works not he?) 'Juan quiere trabajar ¿uo?'

b. Joln will work, won't he? (y no: *......., doesn't he?) 'Juan va a trabajar ¿no?'

Basándose en estos argumentos, Chomsky ha demostrado creo que de un mọdo concluyente que está justificado establecer la distinción entre 'modals' y verbos.

2.2.2. Ė̀n alemán hay seis verbos que tradicionalmente se dicen 'modales': werden 'ir a', wollen 'querer', sollen 'deber', müsscn 'deber', mogen 'poder', dïrfon 'poder' 1.

Bicrwisch (Ig68), como últimamente Jenkins (I972), ha estudiado las propiedades sintácticas de estos verbos y llega a la conclusión de que no hay argumentos sintácticos que permitan aislar este grupo.

Primero, los verbos auxiliares haben/sein 'haber' - que si forman parte de AUX en alemán - puedẹ preceder o seguir a los verbos modalcs:

(13)a. Peter wird einen Ball gesucht haben wollen (werden + wollen + haben + suchen)

'Pedro va a querer haber buscado un balón'.

b. F'eter wird einen Ball suchen gewollt haben (werden + haben + wollen + suchen)

'Pedro va a haber querido buscar un balón'

Segundo, un verbo modal puede scguir a otro:

(I4)a. Er muss kommen dürfen

'El tiene que poder venir'

b. Er soll kommen dürfen müssen

'El debe tener que poder venir'

1 Las glosas de los verbos modales alemanes sólo tienen valor aproximativo. Is inposible dar con equivalencias exactas. 
Nótese además que no hay orden fijo de los verbos modales entre ellos.

Tercero, no hay reglas de transformación que se aplican a los verbos modales y no a los demás.

Basándose en estos argumentos, Bierwisch concluye que en alemán no hay 'modals'.

2.2.3. Ruwet (rg68:185 ss.) se sirve de los mismos argumentos que Bierwisch para demostrar que pouvoir 'poder' y devoir 'deber', los únicos verbos del francés que podrian considerarse como modales, no son auxiliares. Asl avoir 'haber' puede tanto seguir como preceder a devoir.

(15)a. elle doit avoir compris tes paroles

'ella debe haber comprendido tus palabras'

b. elle a du comprendre tes paroles

'ella ha debido comprender tus palabras'

Ademas de éstos, Ruwet se sirve de otro argumento, a saber el hecho de que pouvoir y devoir adiniten a la vez la negación ne ... pas 'no' con ellos mismos y con el verbo que sigue a ellos.

(I6) elle ne peut pas ne pas avoir compris tes paroles 'ella no puede no haber comprendido tus palabras'

Este argumento se basa en la hipótesis de que no se admite más de una sola negación en cada oración simple ${ }^{1}$. Por lo tanto, si decimos que, en (I6), peut es elemento auxiliar, hay dos negaciones en la misma oración simple, lo cual va en contra de una hipótesis bien fundamentada. De alú que sea preferible analizar a (16) diciendo que es oración compuesta, con dos verbos independientes.

2.3.0. Supougamos que, en español, haber más participio pasado, y estar más gerundio son elementos auxiliares: se pueden aducir los siguientes argumentos sintácticos en pro de esta hipótesis.

Primero, no se puede cambiar el orden: haber - estar - V.

(I7)a. Juan ha estado cantando

b. * Juan está habiendo cantado

1 Véase Klima (1964). Toáñez (I972) es un estudio de la negación en espaũol. 
Segundo, estando presente tanto haber como estar como V, no se admite más de una sola negación, la cual está en posición fija delante de haber.

(18)a. Juan no ha estado cantando

b. *Juan no ha no estado cantando

c. *Juan ha estado no cantando

Por lo tanto haber y estar pueden ser considerados como verbos auxiliares, es decir, niembros de AUX, aunque la prueba no es concluyente (véase el apartado 2.5 ).

2.3.I. Veamos ahora si los verbos perifrásticos son miembros del constituyente AUX. Basándome en las discusiones más representativas acerca de este problema ${ }^{1}$, he subdividido los verbos perifrásticos en sicte grupos ${ }^{2}$.

2.3.I.I. Il primer grupo consta de: poder, soler, saber, deber; estos son los que se dicen tradicionalmente 'modales'. Algunos de cstos cuatro verbos pucden tanto preceder como seguir a otros del mismo grupo, sin que se descubra un orden de sucesión básico. Con esto no queremos dar a cntcnder que todas las combinaciones sean aceptables:

(rg)a. Juan debe saber nadar, si es marinero

b. *Juan sabe deber nadar, si se hunde el buque

c. los marineros no suelen saber nadar

d. *los mineros saben poder trabajar en circunstancias muy malas

Los cuatro verbos tienen la libertad de colocarse con haber y con no, tanto si estos elementos les preceden como si les siguen:

(20)a. Juan no ha podido terminar el trabajo

b. Juan puede no haber terminado el trabajo

c. Juan no puede no saber de eso

1 RAE; Gili Gaya; Bello; Roca Pons; Körner; Seco; Bouzet; Ramsey; Lenz; Alonso/Urẽ̃a; Coste/Redondo; Stockwell/Bowen/Martin.

- Téngase en cuenta que no importa cómo se subdividen los verbos perifrísticos. Aqui sólo se trata de una division arbitraria. Hemos hecho caso omiso de algunos verbos que se dicen auxiliares: seguir, andar, ..., los cuales exigen la forma del gerundio del verbo siguiente, y los que requieren un participio pasado, como tener, dejar, ser, llevar, estar,... En un principio, seguir, etc., sustituye a estar ; .tener, etc., ofrecen problemas especiales que dejaremos de un lado. 
(2r)a. Juan no ha debido correr todo el camino

b. ?? Juan debe no haberse mojado los pies

c. ??Juan no debe haberse informado de ello

(22)a. el atleta suele haber corrido las diez millas antes de tomar el desiryuno

b. ?? el anciano ha solido correr cinco quilómetros diarios en su juventud

c. el ministro sucle no darse por aludido en los debates

d. el ministro no stucle darse por aludido en los debates

(23)a. el chico listo sabe haber terminado todos los deberes antes de que comience la función de la noche

$b$. el ilustre politico ha sabido construir un castillo de naipes

c. el gerente sabe no cansarse mucho en su trabajo

$d$. el ingeniero no sabe construir puentes levadizos

Nótese que es diff́cil construir oraciones aceptables en que haber o no sigan a soler y saber. Esto no quita para que se dé la posibilidad, como queda demostrado en (22) y (23).

De todo esto se sigue que los verbos modales no son auxiliares, o sea, que no son miembros de $M$.

2.3.I.2. "Otro grupo de verbos, que, por su parecido "con los verbos modales, se podrían denominar semi-modales, está constituido por querer, pensar, crecr, dcsear, lograr; suponer, etc. Es fácil ver que con todos y cada uno de ellos se pueden construir oraciones aceptables en las que van precedidos o seguidos de haber y no.

Me linito a presentar un solo ejemplo: .

(24)a. Juan no ha querido evitar las discusiones

b. Juan quisiera no haber evitado las discusiones

Por lo tanto, estos verbos no son auxiliares.

2.3.I.3. Exactamente los mismos argumentos valen para que lleguemos a la conclusión de que no son auxiliares los verbos que constituyen c1 tercer grupo: necesitar, parecer, resultar, intentar.

Basta con dos ejemplos:

(25)a. Juan intenta no romper los vidrios

b. el pobrecito ese parece lhaber heredado varios miles de millones. de dólares. 
RFE, LVII, 19745 EN TORNÓ A LOS VERBOS PERIFRASTICOS DEL ESPAÑOL.

2.3.I.4. Il grupo de los verbos perifrásticos que significan obligación se compone de: tener que, tener de, haber de, hay que. Aunque muchas veces sea un poco violento introducir haber o no después del verbo obligativo, no se pueden rechazar todas las oraciones como:

(26)a: usted tendrá que noo immiscuirse cn los asuntos de las personas mayores

b. Los obreros habian de haber delatado al soplón

c. ? ?hay que no dejarse engaũar por las apariencias

Por consiguiente, estos verbos difícilnuente se pueden admitir como clementos auxiliares.

2.3.I.5. - Los verbos perifrásticos que significan iniciación del acto denotado por el verbo siguiente, como lo son: comenzar, empezar, cchar(se), ponerse, pasar, todos seguidos de $a$, muchas veces no permiten que se introduzea $n 0$ o haber entre ellos mismos y el verbo siguicnte:

(27)a. cl monarca comenzó a no levantarse de mañana

b. ??la noticia comenzaba a haber perdido todo interés

c. ??el jovencito se puso a no hacer más que tocar la guitarra

Auncue en algunos casos sea posible consitruir oracioncs de este tipx, las cuales, dada una situación adecuada son aceptables, en la mayoría de los casos son inaceptables.

Por lo tanto, comenzar, etc. podrían considerarse como elementos auxiliares.

2.3.r.6. Los verbos perifrásticos iterativos volver y tornar, seguidos de $a$, se comportan de la misina manera que comcnzar, etc., como se pucde ver en:

(28)a. ?? el enfermo volvió a no comer

b. ??el niño mimado tornará a haber sido débil de salud

Por lo tanto, en este momento no hay por qué negarles el ser auxiliares.

2.3.I.7. Los verbos perifrásticos terminativos: acabar, dejar, concluir, terminar, todos seguidos de de, presentan las mismas caracteristicas que comenzar, etc.: 
(29)a. ??el payaso acaba de haber bailado en la cuerda

b. ??el plan de estudios acaba de no estimular a la mayorla de los estudiantes

Una vez más, podemos concluir que no hay por qué negarles a estos. verbos perifrásticos el ser auxiliares.

2.3.2. Las pruebas que acabamos de aplicar son muy sencillas. De ellos se podria deducir que AUX contiene no solamente TMP, haber, estar, sino también una clase de verbos auxiliares $\mathrm{M}_{1}$. la cual consistiria de comenzar, etc., volver, etc., y terminar, etc. Sin embargo, serfa una conclusión errónea, ya que las pruebas sólo valen para demostrar que ciertos verbos perifrásticos no son auxiliares. No podemos demostrar, con sólo aplicar estas pruebas, que otros verbos perifrásticos necesariamente han de tomarse por auxiliares. Para ello, se necesita evidencia positiva, es decir, hay que justificar la distinción tajante entre los verbos no-auxiliares y los auxiliares.

2.4.0. Comparemos el comportamiento sintáctico de un verbo cualquiera como trabajar con el de haber, estar y comenzar.

2.4.I. En frases interrogativas que comienzan con un pronombre interrogativo como qué, quien, ... es obligatorio trasladar el sujeto a otra posición, de forma que puede venir a encontrarse detrás del primer verbo. Asi, se nota que (30)a. es incorrecta, mientras que (30)b. es correcta.

(30)a. * ¿dónde Juan trabaja?

b. ¿dónde trabaja Juan?

Bajo las mismas condiciones, el sujeto también puede posponerse a estar y comenzar, pero no a haber:

(3r)a. * ¿dónde ha Juan trabajado?

b. ¿dónde está Juan trabajando?

c. ¿dónde comienza Juan a trabajar?

Con lo dicho arriba no queremos dar a entender que Juan esté en la posición preferida en las oraciones de (3I): sí mantenemos que Juan se encuentra en una de las posiciones sintácticamente admisibles.

2.4.2. En oraciones negativas, no precede al verbo, como también precede a haber, estar, comenzar: 
(32)a. Juan no trabaja

b. Juan no ha trabajado

c. Juan no está trabajando

d. Juan no comienza a trabajar

2.4.3. La posición de los pronombres clíticos respecto a los verbos no difiere de su posición respecto a haber, estar, comenzar: los preceden si hay desinencias temporales; siguen a ellos si hay desinencia de infinitivo o gerundio y no se admiten si hay desinencia de participio pasado.

(33)a. se lo entregó vs. entregárselo

b. se lo ha entregado vs. habérselo entregado

c. se lo está entregando vs. estárselo entregaudo

$d$. se lo comienza a entregar vs. comenzárselo a entregar

2.4.4. Hay adverbios como ya, siempre, que normalmente se ponen cn posición preverbal: también se anteponen a haber, estar, comenzar:

(34)a. Penélope siempre teje la tela

b. Penélope siempre ha tejido la tela

c. Penélope siempre está tejiendo la tela

d. Penélope siempre comienza a tejer la tela.

2.5. Hemos enumerado algunos fenómenos sintácticos que hubicran podido proveernos de argumentos para aislar un grupo de elementos auxiliares. No damos con uingún argumento positivo. Esto inılica que las complicaciones que surgirian por distinguir un grupo asl, como, por ejernplo, la necesidad de complicar $T$-afijo, wo están motivadas de ninguna manera. Es más, cada vez que intentásemos explicar algún fenómeno sintáctico como los mencionados ell 2.4.I.-2.4.4. mediante una regla de transformación, tendríamos que especificar que la regla se aplicaba a la clase constituida por V, haber, estar, MI, lo que solamente en el caso de haber parece justificado.

En cada una de las transformaciones, pues, tendrímos que distinguir una clase $\mathrm{v}=\mathrm{V}$, estar, haber, $\mathrm{MI}$, sin que, hasta ahora, hayamos podido descubrir regla alguna que mencione sólo $\mathrm{V}$, o sólo estar, haber, M.

La conclusión que se impone es que, sintácticamente hablando, no hay vetbo auxiliar en español, si no es haber. De esta manera simplificamos las reglas de rescritura, y también las reglas de transformación. 
Como sabemos muy poco del componeute; semántico; no se sabe si éste queda afectado o no por la simplificación :del componente sintáctico,

3. Las oraciones completivas del español y los verbos perifrásticos.

3.0. Una vez descartada la hipótesis de que hay verbos auxiliares en español, intentaremos esbozar una teoría general de las oraciones completivas en español, que en parte explicará las caracteristicas de los verbos perifrásticos.

3.I.o. El español tiene varios tipos de oraciones subordinadas: por las desinencias verbales podemos distinguir cuatro. .

Primero, hay oraciones subordinadas cuyo verbo tiene desinencia que desigua a la vez número, persona, tiempo y modo. A estas oraciones las denominaremos oraciones temporales. Haremos caso omiso de las diferencias que se hallan entre oraciones temporales con el verbo en modo indicativo y con el verbo en modo subjuntivo ${ }^{1}$.

Segundo, hay oraciones subordinadas cuyo verbo tiene la desinencia $r$ del infinitivo.

Tercero, hay oraciones subordinadas cuyo verbo tiene la desinencia ndo del gerundio.

Cuarto y último, hay oraciones subordinadas cuyo verbo tiene la desinencia do del participio pasado.

Sirvan de ejemplo:

(35)a. Siempre he dicho que no cs redonda la tierra (oración subordinada temporal con el verbo en modo indicativo)

b. Conviene que aplaces tu vuelo a la luna (oración subordinada temporal con cl verbo en modo subjuntivo)

c. Ies he prometido volver antes de las diez (oración subordinada de infinitivo)

d. Los alumnos seguían mostrándose hostiles (oración subordinada de gerundio)

e. La actriz tenía encantado al priblico (oración subordinada de : participio pasado).

3.I.I. Supongamos que cada oración, sea principal o subordinada, va precedida, en la cstructura subyacente, de un elemento que podemos

1 Para un intento de explicación del subjuntivo, véase Schroten (1975). 
llamar completivador ${ }^{1}$ (abreviado: COMP). La primera regla de rescritura del español será:

$\mathrm{R}$ (vii) $\mathrm{S} \longrightarrow \mathrm{COMP} \quad \mathrm{NP} \quad \mathrm{VP}$

Supongamos además que COMP se rescribe:

$\mathrm{R}$ (viii) $\mathrm{COMP} \longrightarrow \ldots^{2} \quad \mathrm{AUX}$

y que AUX se rescribe:

$\mathrm{R}$ (ix) $\quad \mathrm{AUX} \longrightarrow$ TMP, r, ndo, do

En oraciones independientes, AUX suele ser TMP, aunque hay casos de oraciones independientes de infinitivo y de gerundio:

(36)a. Juan to dijo (oración independiente temporal)

b. ja callarl (oración independiente de infinitivo)

c. andando! (oración independiente de gerundio)

Que yo sepa no hay oraciones independicutes de participio ${ }^{3}$.

Hay cuatro categorias sintácticas que permiten oraciones completivas, a saber, los verbos, los sustantivos, los adjetivos y las preposiciones. Todas estas categorias permiten oraciones completivas temporales:

(37)a. te convenci de que ios criterios formales no sirven para nada (verbo)

$b$. tu opinión de que los critcrios formales no sinven para nada me pone muy triste (sustantivo)

c. es muy perverso que pienses que los criterios formales no sirven para nada (adjetivo)

d. sin que te hubiesen saludado, siguieron adclante (preposición)

Todas permiten oraciones completivas de infinitivo:

(38)a. te invité a acompañarme a la final de copa (verbo)

b. tu decisión de abandonar el proyecto me alegra (sustautivo)

c. es convenienic conectar primero los cabos (adjetivo)

d. sin decirte adiós, huyó el pistolero (preposición)

Todas permiten oraciones completivas de gerundio:

1 Para una argumentación, véase Chomsky (1973) y Bresnan (1970).

- Como no estudiarcmos más que AUX, haremos caso omiso de los demás elementos de COMP.

- No sé cómo explicar este hecho. Se nue sugicren las siguientes: je ancluidol - jarrcgladol, etc. 
(39)a. Goya pintó a los soldados fusilando a sus adversarios (verbo)

b. este es el cuadro ${ }^{1}$ de los soldados fusilando a sus adversarios (sustantivo)

c. mira los campos de Castilla evocativos 2 de ejércitos pasando por ellos (adjetivo)

d. en llegando César, salió Bruto (preposición)

Solamente unos pocos verbos permiten oraciones completivas de participio:

(40)a. César dejó redactada su gramática (verbo)

b. ???

c. ???

(sustantivo)

d. ???

Que yo sepa, no hay sustantivos, ni adjetivos, ni preposiciones que permitan oraciones completivas de participio?.

1 Me parece que, en estos casos, la forma de gerundio depende del sustantivo. Como las gramb́ticas suelen decir que, en esta construcción, la oración con el verbo en forma de gerundio no equivale a una oración relativa, sino que más bien significa el acto a que se refiere la oración entera, este análisis tiene evidentes ventajas. Nótese que hay una clase natural de sustantivos parecidos como: cuadro, pintura, descripcion, relato, cuento, historia. Así se explica que los titulos de cuadros presenten el verbo independiente en forma de gerundio: es que se subentiende la palabra cuadro. Por ejemplo, supongo que las ranas pidiendo rey quiere decir: 'este es el cuadro de las ranas pidiendo rey'. I,as caracteristicas de esta construcción enigmática creo que se explican de un modo natural, si se adopta este punto de vista.

2 No tengo la certeza que se dé esta construcción. Sin embargo, no parece descabellado suponer que los adjetivos evocativo, conmemorativo, descriptivo a veces se combinan con oraciones completivas de gerundio que dependen de ellos. No he podido dar con otros adjetivos de esta clase. Ni pintoresco, ni novelesco, ni otros adjetivos relacionados con los sustantivos enumerados en la nota precedente permiten oraciones completivas.

- Como tampoco hay oraciones independientes de participio pasado, se puede sostener que los verbos seguidos de participio pasado si son awxiliares. Hay otro argumento: los verbos con la desinencia de participio pasado no permiten la atracción de pronombres clíticos. Además de esto, deben ser analizados como adjetivos en las construcciones de carácter pasivo.

No sé cómo explicar estas peculiaridades del participio; por ahora, tiene poco interés sostener una u otra opinión. Hasta que sea presentada una hipótesis que explique los fenómenos mencionados, dejaré abierta la cuestión. Tal vez sea válido cl punto de vista de Alarcos; éste sosticne que no hay construcción pasiva en español y que se debe interpretar la pasiva cowo wa construcción de cópula más adjetivo (Alarcos (1970: cap. VII)). 
RIE, LVII, 1974-5 EN TORNO A LOS VERBOS PERIFRASTICOS DEI. ESPAÑOL

3.I.2. Una vez establecida la tipologia general de las oraciones completivas, pasamos a establecer unas pocas precisiones.

Primero, hay que poner que delante de cada oración completiva temporal. Fácilmente se descubren unas pocas excepciones a esta regla general: por un lado, hay (tener) + que + verbo en infinitivo; por otro lado, si el verbo dominante es rogar, etc., se puede no poner que delante de la oración completiva temporal.

Segundo, hay que poner AUX detrás del verbo correspondiente. Proponemos dos reglas de transformación:

$\mathrm{T}$ (ii) 'T-inserción de QUE (obligatoria)

$$
\frac{\mathrm{X}-\mathrm{V}-\ldots}{\mathrm{I}} \frac{\ldots \mathrm{TMP} \ldots-\mathrm{V}-\mathrm{Y}}{2} \longrightarrow \text { I, que }+2
$$

T(iii) T-atracción de $A U X$ (obligatoria)

$$
\frac{X}{I}-\frac{A U X}{2}-\frac{\ldots}{3}-\frac{V}{4}-\frac{Y}{5} \longrightarrow I, \varnothing, 3,4+2,5
$$

Condición: ... no contiene V

T(iii) debe aplicarse después de $T$ (ii).

I a formulación y especificación de las dos reglas es provisional y sirve sólo para precisar un poco las operaciones sintácticas.

3.I.3. Is necesario suponer que cada palabra que admite oraciones completivas, especificará el tipo de oraciones completivas que admite. Así, decir admite oraciones completivas temporales y de infinitivo; qucrer, idem; atreverse, solamente admite oraciones completivas de infinitivis, pintar sólo de gerundio ${ }^{1}$. Todo esto no tiene nada de nuevo, y se presupone en todos los estudios que se han ocupado del asunto.

3.2.o. Ahora bien, una de las peculiaridades de las oraciones completivas de infinitivo y de gerundio consiste ell que éstas, en la estructura superficial, carecen de sujeto:

(4x)a. El pueblo obligó al monarca a dimitir

b. Lil pucblo no quería aceptar la propuesta

c. Los ministros siguieron discutiendo el problema

1 Nótese que la tcoría aquí expuesta difiere radicalmente de la adoptada por MLolina Redondo (1971): 
En la gramática tradicional suele decirse que el sujeto de la oración completiva se subentiende. En la teoría transformacional, se supone que cada oración tiene sujeto en la estructura subyacente. Hay varias maneras de dar una interpretación formal tanto a la intuición gramatical tradicional como a la exigencia teórica.

Tomando como ejemplo la oración (4r)a., se pueden postular varias estructuras subyacentes que captan las intuiciones de los gramáticos:

(42)a. el pueblo obligó [s el monarca - a dimitir]

b. el pueblo obligó al monarca [sel monarca - a dimitir]

c. el pueblo obligó al monarca $\left[{ }_{s}\left[{ }_{\mathrm{NP}} \Delta\right]^{2}-a\right.$ dimitir $]$

$\mathrm{Si}$ (42)a. fuese la estructura subyacente de (4I)a., habria que formular una regla de transformación que atrajese el sujeto de la oración complemento al verbo dominante, convirtiéndolo en objeto de obligar. Si (42)b. fuese la estructura subyacente de (4I)a., habría que formular una regla de transformación que suprimiese el sujeto de la oración complemento, sustituyéndolo por cero. Si (42)c. fuese la estructura subyacente de (4r)a., habría que formular una regla de interpretación semántica que especificase que $\left[{ }_{\mathrm{NP}} \Delta\right]$ recibe la misma interpretación semántica que el monarca ${ }^{2}$, o sea, el objeto del verbo dominante.

Es imposible saber de antemano cuál es el método más apropiado. Para nuestro propósito, las consecuencias emṕricas que se siguen de ellos son las mismas, lo que equivale a decir que son variantes notacionales. Sin embargo, la más generalizable es la última ${ }^{3}$. Por lo tanto, investigarenos las posibilidades de explicación que ofrece.

3.2.I. Essbocemos primero cómo se interpreta $\left[{ }_{\mathrm{NP}} \Delta\right]$. Postulamos una regla de interpretación semántica para $\left[{ }_{\mathrm{NP}} \Delta\right]$ :

$1 \Delta$ es el llamado 'comodin' (inglés 'dummy'). Para una justificación del uso de $\Delta$ véase Chomsky (1965) y sobre todo Jackendoff (1971). El comodin es algo ast como un pro-elemento (pro-nombre, o pro-sintagma nominal, en este caso) de forma cero, es decir, sin representación fonética.

- Véase Jackendoff (I97r).

s Ampliando un poco la interpretación semántica, se podría proponer que:

(i) [NP $\Delta]$ recibe la misma interpretación semántica que otro NP; (se dice que hay correferencia entre NP $\mathrm{y}\left[\mathrm{NP}_{\mathrm{P}} \Delta\right]$, o que NP y $\left[\mathrm{NP}_{\mathrm{P}} \Delta\right]$ son correferentes);

(ii) si $\left[\mathrm{NP}_{\mathrm{P}} \Delta\right]$ no recibe interpretación semántica mediante (i), se interpreta como 'uno', bajo ciertas coudiciones estructurales - isi hay infiwitivo o gerundio?-;

(iii) si $\left[{ }_{N P} \Delta\right]$ sigue sin interpretación, la oración en que se halla es anómala. 
S(i) Sea una estructura de la forma:

$\ldots \mathrm{NP} \ldots\left[\mathrm{s} \ldots-\mathrm{I}-\left[_{\mathrm{NP}} \Delta\right]-\mathrm{V}-\ldots\right]$

In ella $\left[{ }_{N P} \Delta\right]$ recibirá la misma interpretación que $N P$, bajo cicrtas condiciones ${ }^{1}$.

No especificarcmos las condiciones: baste con decir que son condiciones estructurales y lexicales. Por ejemplo, NP no puede encontrarse cn uula oración más 'baja' que $\left[_{N \mathrm{P}} \Delta\right]$ ] (en: vi a los chicos que les estaban diciendo piropos a las chicas desaparecer por cntre la multitud, el sujeto de desaparecer no es las chicas); el verbo de la oración en que se encucntra NP debe especificar cuáles de los NP pueden servir de antecelente de $\left[{ }_{N I} \Delta\right]$ (en: yo lcs mandé callar vs. yo les prometi callar se ve que mandar permite al objeto que sirva de antecedente, y que prometer permite al sujeto que sirva de antecedente) ${ }^{2}$.

Hay varios problemas importantes que requieren una solución. Entre otros, cabe preguntarse si todas las oraciones completivas de infinitivo tienen sujeto $\left[{ }_{\mathrm{N}} \Delta\right]$, o si hay oraciones cuya cstructura subyacente es análoga a (42)a. Piénsese en oraciones como: vi a los niños correr, hizo trabajar a los alumnos, etc. No nos ocuparemus dic este problema. Solamente investigaremos algunas de las projicdades sintácti-

- cas y scmánticas de los verbos perifrásticos mediantc una comparación con verbos no-perifrásticos que requieron una oración completiva de infinitivo.

3.3.0. Sca comenzar un verbo perifrástico y quercr un verbo no-perifrástico, y compárense las oraciones siguientes:

(43)a. Juan comienza a atacar a Pedro

b. Pedro comienza a ser a.tacado por Juan

(44)a. Juan quiere besar a María

b. María quicre ser besada por Juan

La relación que hay entre (43)a. y (43) b. parece ser is misma que existc entre (44)a. y (44)b.: las oraciones b. son los equivalcntes pasivos de las oraciones a. Sin embargo, hay una diferencia. Imaginémonos una si-

1 Ia formulación necesariamente ha de ser torpe a fin de evitar rodcos inutiles. Sin cmbargo lo que va a continuación sigue sicndo válido aún cu una formulación, digamos, mnás sofisticada.

2 Véase Jackendoff (197I). 
tuación que está correctamente descrita por (43)a.; en este caso es inconcebible que (43)b. no describa de una manera apropiada la misma situación. Imaginémonos ahora otra situación que está correctamente descrita por (44)a. En este caso no hay ninguna garantía de que (44)b. describa de una mancra apropiada la misma situación. Es más: tỏdos sabemos por expericncia que existe mucha distancia entre la situación (44)a. y la situación (44)b. Dicho de otro modo, si (43)a. es verdad, (43)b. también es verdad. Si (43)a. es falso, (43)b. también lo es. Pero si (44)a. es verdad, (44)b. muy bien puede ser falso, y al revés. Eso se ve muy fácilnente introduciendo la conjunción adversativa pero entre (43)a. y (43)b., (44)a. y (44)b., y añadiendo a (43)b., (44)b. la negación no:

(43)c. *Juan comienza a atacar a Pedro, pero Pedro no comienza a ser atacado por Juan

(44)c. Juan quiere besar a Maria, pero María no quiere ser besada por Juan

Dicen los lógicos que (43)c. representa un juicio contradictorio y (44)c., no. Esta diferencia que hay entre comenzar y querer es muy notable y requiere una explicación. Volveremos luego al asunto.

3.3.I. Los verbos que podrfan llamarse meteorológicos, como llover, granizar, son admisibles como complemento de comenzar, y no lo son como complemento de querer:

$\left(43^{\prime}\right)$ a. comienza a llover

b. *quiere llover (sólo es aceptable en dialectos en que significa: suele 1lover)

Pero el fenómeno es más general: si un verbo admite cierto NP como sujeto, también es aceptable la oración en que comenzar precede al verbo; esto no se cumple si sustituímos comenzar por querer:

$\left(44^{\prime}\right) a$. *la piedra viene

b. la piedra viene abajo

c. *la piedra comienza a venir

d. la piedra comienza a venir abajo

e. *la piedra quiere venir

f. *la piedra quiere venir abajo 
Dicho de otra manera, no hay relación entre el sujeto superficial de comcnzar y el propio verbo comenzar, sino que la hay entre el sujeto superficial de comenzar y el verbo del complemento de comenzar. De otra manera se comporta quever: este verbo requiere un sujeto animado, es decir, un sujeto capaz de ejecutar actos de volición. Las restricciones de compatibilidad entre el sujeto y el verbo probablemente sc explican preferentemente por medio del componente semántico: parece ser propiedad semántica de piedra el que no sea capaz de ejecutar actos de volición.

3.3.2. Hemos descubierto que comenzar no tiene relaciones semánticas con cl sujeto de la estructura superficial. En esto se parece mucho a las desinencias verbales.

Intentemos formarnos una idea acerca de lo que tiene que explicar el componente semántico en este caso y consideremos de nuevo (43), (44), que repetimos para facilitar el cotejo:

(43)a. Juan comicnza a atacar a Pedro

b. Pedro comienza a ser atacado por Juan

(44)a. Juan quiere besar a María

b. María quiere ser besada por Juan

$\Lambda$ fin de interpretar correctamente (43)a. y (43) b., tenemos que especificar (que el significado de comenzar domina sobre el significado de Juun ... alacar a Pedro cn (43)a., y de Pedro ... ser alacado por Juan en (4.3)b. lis decir que, en la interpretación semántica, comenzar toma un solo argunnento $S$, la cual, por lo visto, consiste en el sujeto superficial de comenzar más el complemento superficial de comenzar. De este modo se explica la equivalencia lógica de (43)a. y (43)b., ya que las dos oraciones se interpretan como: Comenzar (S), y como: Comenzar (S'), respectivamente, siendo $\mathrm{S}=$ Juan atacar a l'edro y $\mathrm{S}^{\prime}=$ Pedro ser atacado por Juan. Es conocida la equivalencia lógica de $\mathrm{S}$ y $\mathrm{S}^{\prime}$; de ahí que: Comenzar $(\mathrm{S})=$ Comenzar (S'). Por lo que se refiere a (44)a. y (44)b., querer parece tomar dos argumentos, NP y S, siendo NP el sujeto superficial de querer y S su complemento. Semánticamente hablando, (44)a. equivale a: Querer (NP, S) y (44)b. a: Querer (NP', S'). Aunque $S=S^{\prime}$, no es verdad que $N P=N P^{\prime}$. De ahi que, dadas las fórmulas: Querer (NP, S), Querer (NP', $\left.\mathrm{S}^{\prime}\right), \mathrm{S}=\mathrm{S}^{\prime}, \mathrm{NP}$ \# $\mathrm{NP}^{\prime}$ se siga que: Querer (NP, S) tt Querer (NP', S').

Si la estructura subyacente en que se inserta comenzar fuese dife- 
rente de aquella en que se inserta querer, las diferencias semánticas se basarian en diferencias sintácticas.

En efecto, se explican las diferencias semánticas entre (43) y (44), postulando que (43) se deriva de (45)a. y (44) de (45)b.

$(45)$ a.
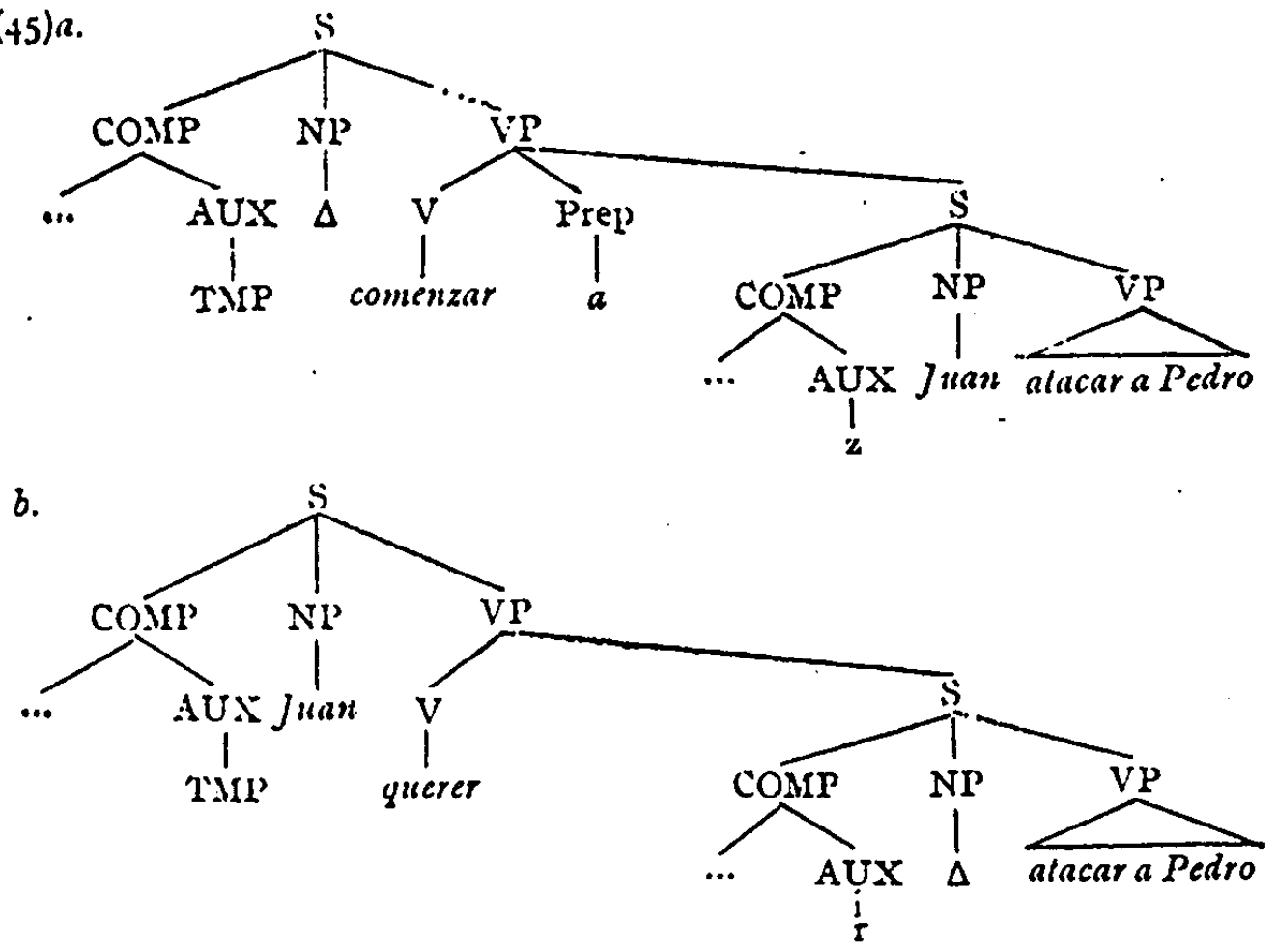

Aqui se necesita una regla de transformación, que llamaremos T-Elevación del Sujeto (abrevindo: T-e. s.).

$\mathrm{T}$ (iv) T-elcicción del sujeto

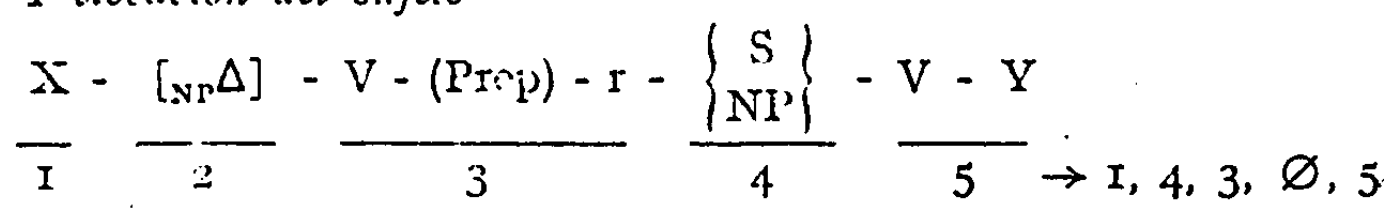

En breve: paliabras: si hay una cstructura en que se distinguen: un sujeto cero $\left(\left[_{{ }_{\mathrm{p}}} \Delta\right]\right)$, un $\mathrm{V}$, más una oración completiva de infinitivo, se sustituyc $\left[{ }_{x p} \Delta\right]$ por el sujeto de la oúción completiva, el cual puede ser NP o $S$.

En (45) b., suponemos que $S(i)$ se aplica, recibiendo $\left[{ }_{N P} \Delta\right]$ la misma interpretación semántica que el sujeto del $\mathrm{V}$ de cuyo complemento $\left[_{\mathrm{NP}} \Delta\right.$ ] es el sujeto.

Como es necesario postular $T(i v)$, si adaptamos este análisis, se complica el componente sintáctico. Pcro esta complicación del componente sintáctico está compensada probablenente por una simplificación del componente semántico, dado el hecho de que se obtiene la 
generalización de que cada uno de los argumentos semánticos corresponde con un solo constituyente no discontinuo de la estructura subyacente. Como comenzar tiene sujeto cero, es lógico que solamente la oración completiva sirva de argumento semántico. De manera similar querer tiene dos argumentos semánticos que corresponden con dos constituyentes sintácticos de la estructura subyacente.

Nótese que el análisis que hemos propuesto tiene cierta ventaja conceptual, y que la complicación sintáctica probablemente está compensada por una correspondiente simplificación del componente semántico. Nos resta demostrar que hay argumentos sintácticos en pro de la solución que acabamos de proponer.

3.4.0. Se enumerarán a continuación las diferencias sintácticas que lay entre comenzar y querer.

3.4.I. Primero, el sujeto superficial de comenzar puede ser una oración sujeto; csto no lo permite quever:

(46)a. que no sepas nunca qué hacer me fastidia

b. me fastidia que no sepas nunca qué hacer

c. que no sepas nunca qué hacer comienza a fastidiarme

d. comienza a fastidiarme que no sepas uunca qué hacer

$e$. *que no sepas uunca qué hacer quiere fastidiarme

f. *quiere fastidiarme que no sepas uunca qué hacer

3.4.2. Segundo, en las oraciones impersonales y pasivas con se, que hemos denominado seudo-reflejas, el se seudo-reflejo ${ }^{2}$ no puede seguir a un infinitivo con querer, y sí con comenzar:

(47)a. se vende la casa

b. comienza a venderse la casa

c. se comienza a vender la casa

d. *quiere venderse la casa

e. se quiere vender la casa

3.4.3. Tercero, en las oraciones seudo-reflejas hay concordancia o no cntre comenzar y el objeto del verbo en infinitivo; nunca hay concordancia entre querer y el objeto del verbo en infinitivo.

1 Para un estudio iuás detallado, véase Schroten (1972). 
(48)a. se comienzan a vender las casas

$b$. se comienza a vender las casas

c. *se quieren vender las casas

$d$. se quiere vender las casas.

3.5.0. Dado el heclio que se han descubierto tres diferencias sintácticas entre comenzar y querer, cabe preguntarse si éstas se pueden explicar mediante las diferencias en las estructuras subyacentes y las transformaciones que acabamos de proponer.

3.5.I. De una manera muy trivial se explica el que comenzar admita oraciones sujeto en la estructura superficial, y querer no: T-e. s. menciona las oraciones sujeto en la descripción estructural, de modo que se debe elevarlas. Querer, por el contrario, sólo admite sintagmas nominales como sujeto, de modo que no hay nada que explicar por lo que se refiere a la inaceptabilidad de (46)e., f.

3.5.2. La oración (47)a. se explica mediante una regla de transformación, T-seudo-reflejo, dada la estructura subyacente:

(49) $\left[{ }_{N P} \Delta\right]$ - vende - la casa

T-seudo-reflejo es algo así como ${ }^{2}$ :

$\mathrm{T}$ (v) T-seudo-reflejo

$$
\frac{[\mathrm{NP} \Delta]}{\mathrm{I}}-\frac{\mathrm{V}}{2}-\frac{\mathrm{NP}}{3} \longrightarrow 3,2+s e
$$

En esencia, T-seudo-reflejo tiene el efecto de sustituir el sujeto $\left.{ }_{\mathrm{NP}} \Delta\right]$ por el objeto y de introducir a la vez el pronombre reflexivo se. Ahora bien, si (47)a. es complemento de comenzar y de querer con sujeto $\left[{ }_{\mathrm{NP}} \Delta\right]$, obtenemos las siguientes estructuras subyacentes de (47)b.-d.:

(50)a. $\left[{ }_{\mathrm{NP}} \Delta\right]-$ comenzar $-\left[{ }_{\mathrm{s}} \mathrm{r}-\left[_{\mathrm{NP}} \Delta\right]-\right.$ vender $-\mathrm{la}$ casa $]$

b. $\left[{ }_{N P} \Delta\right]-$ querer $-\left[{ }_{8} \mathrm{r}-\left[{ }_{N P} \Delta\right]-\right.$ vender $-1 a$ casa $]$

Apliquemos T-seudo-reflejo en las oraciones subordinadas de (50)a., b. y el resultado será:

1 Una formulación y argumentación mús detallada se hallan en Schroten (1972). 
(5I)a. $\left[{ }_{\mathrm{Nr}} \Delta\right]-$ comenzar $-{ }_{\mathrm{s}} \mathrm{r}-1 \mathrm{a}$ casa - vender $\left.+\mathrm{se}\right]$

b. $\left[{ }_{N D} \Delta\right]-$ querer $-\left[{ }_{s} \mathrm{r}-\right.$ la casa - vender + se $]$

No hay problemas con (5I)a.: el sujeto derivativo la casa debe ser elevildo; de este modo se explica que se esté afijado a vender cn (47) b. I a uración (47)c. se genera de la misma manera que (4S) a.: véase (52). Por el contrario, en $(5 \mathrm{I}) \mathrm{b}$. es inaplicable la regla de interpretación semántica, ya que el sujeto del complemento de querer no es [NT $\Delta$ ], sino la casa.

Ya que querer exige que se aplique la regla, (47)d. no recibe una interpretación semántica adecuada. De alni que sea inaceptable.

3.5.3. Lo dicho arriba explica a la vez el paradigna de (48). En (48)a., las casas ha sido elevado y sirve de sujeto de comcnzar; pero no hay tal cosa con quever como acabamos de demostrar. La posición de se delante de comcnzar es fenómeno aparte que requiere una explicación más generalizada; limitémonos a decir que hay una regla de 'movimiento de cliticos' que traslada los clíticos hacia la izquierda bajo ciertas condiciones estructurales que están satisfechas en (48)a. La derivación de (48)a. se explica así:

(Estructura subyacente:) $\left[{ }_{N P} \Delta\right]-$ comenzar $-\left[{ }_{s}{ }^{T}-\left[{ }_{N P} \Delta\right]-\right.$ - vender - las casas] $\longrightarrow$ T-seudo-reflejo $\longrightarrow$

(Estructura intermedia I:) $\left[{ }_{N P} \Delta\right]-$ comenzar $-\left[{ }_{s}-\right.$ las casas - vender + se $] \longrightarrow$ T-e. s. $\longrightarrow$

(Estructura intermedia 2:) las casas - comenzar - ${ }_{5} \mathrm{r}-$ vender $+\mathrm{se}] \longrightarrow$ incorporación de $r$ en vender, y concordancia de las casas y comenzar

(Estructura intermedia 3:) las casas comienzan a [vender + se] $\longrightarrow$ T-moviniento de clíticos $\longrightarrow$

(Estructura superficial:) las casas se + comienzan a [vender]

Ya hemos visto cómo, con el verbo querer, la derivación análoga descarrila en cierto momento: por lo tanto, no se puede generar (48)c. Supongamos ahora que T-seudo-reflejo no se aplica en las oraciones subordinadas de (50), y apliquemos, en la oración principal, T-e. s. en (50)a., S(i) en (50)b. El resultado es:

(53)a. $\left[{ }_{\mathrm{Nr}} \Delta\right]-$ comicnza $-[\mathrm{r}-$ vender - la casa $]$

b. $\left[{ }_{\mathrm{NP}} \Delta\right]-$ querer $-\left[\mathrm{r}-\left[_{\mathrm{NP}} \Delta \mathrm{i}\right]-\right.$ vender - la casa $]$ 
El signo [ ${ }_{\mathrm{NP}} \Delta \mathrm{i}$ ] simboliza el comodin que ha sido interpretado. Aplicando T-seudo-reflejo en las oraciones principales de (53)a., b., obtenemos:

(54)a. [svender la casa] - comenzar + se

b. $\left[{ }_{\mathrm{s}}\left[{ }_{\mathrm{NP}} \Delta \mathrm{i}\right]\right.$ vender la casa $]$ - querer + se

En los dos casos, ya que el sujeto del verbo no es un sintagma nominal en plural, el verbo está en tercera persona del singular. Así quedan explicadas las oraciones (47)c., e., y (48)b., d., cuya derivación corre paralela a la de (47)c., e., y eso de una manera intuitivamente no del todo insatisfactoria 1.

3.6. Creenos haber demostrado que comenzar y querer se diferencian sintáctica y semánticamente. A fin de explicar estas diferencias, hemos propuesto varias hipótesis:

(i) comenzar debe ser inscrtado en una estructura subyacente de la forma: $\left[{ }_{N P} \Delta\right]-\ldots-\left[{ }_{s} r-N P-V P\right]$ en la posición de querer debe ser insertado en una estructura subyacente de la. forma: NP - — - $\left[{ }_{s} I-\left[{ }_{N P} \Delta\right]-V P\right]$ en la posición de

(ii) hay una regla obligatoria de transformación llamada T-elevación del sujeto que, en la estructura: $\left.{ }_{\mathrm{NP}} \Delta\right]-\mathrm{V}-\left[{ }_{s} \mathrm{r}-\mathrm{NP}-\mathrm{VP}\right]$ sustituye $\left[{ }_{\mathrm{NP}} \Delta\right]$ por NP.

(iii) hay una regla obligatoria de interpretación semántica, que en la estructura: $N P-V-\cdot\left[{ }_{s} T-\left[{ }_{N P} \Delta\right]-V P\right]$, da a $\left[{ }_{N P} \Delta\right]$ la misma interpretación semántica que NP.

(iv) hay una regla opcional $\mathrm{T}$-seudo-reflejo que transforma las estructuras: $\left[{ }_{N P} \Delta\right]-V-N P-\ldots \rightarrow N P-V+$ se $-\ldots$

1 Obsérvese que (47)c. pernite doble derivación, lo cual implica que la misma estructura subyacente está relacionada con una sola estructura superficial mediaute dos derivaciones distintas. Iss un poco sospechosa esta extraña coincilencia. Obscirvese además que no está claro cómo podemos imponer la condición de que. T-sendo-reflejo no se aplique en la oración subordinada y si en la principal. En realidad, ine parece necesario postular que comenzar puede ser insertado en las dos estructuras especificadas en el apartado 3.6. (i), es decir, que puedeser introducido también en el entorno de quever, derivando (48)b. de la misma manera que (48)d. Todo esto no afecta directamente a la argumentación, como se puede ver fácilmente.

l'or último huelga decir que he dejado de lado la posición del sujeto superficial; se supone que va a encontrarse en posición postverbal en todos los casos. 
Estas cuatro hipótesis, junto con otras (p. ej.: T-movimiento de clíticos, T-concordancia) explican gran parte de las diferencias sintáctica que hablamos observado entre comenzar y querer. Además, parece plau sible que simplifiquen el componente semántico.

Por lo tanto, conclufmos que todas las hipótesis y cada una de ellas están bien fundamentadas, y que hemos demostrado que las peculiaridades de los verbos perifrásticos muy bien pueden explicarse, sin que sea necesario considerarlos como miembros del constituyente AUX.

JAN SCHROTEN

Universidad de Utrecht 


\section{BIBIIOGRAFIA}

AIARCOS II,ORACH, EAIILIO (1970). Estudios do gramdica funcional del español. Madrid. Gredos.

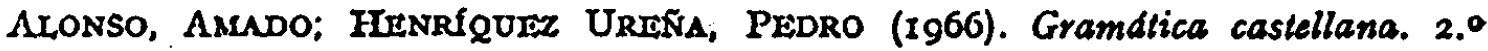
curso. Buenos Aires. Iosada.

BELLO, ANDREs (195I). Gramdtica de la lengua castellana destinada al uso de los americanos. Bogotá (Obras Completas, 5).

BIERWISCH, MLANRRED (1963). Grammatik des deutschen Verbs. Berlin. AkademieVerlag. (Studia Grammatica, 2).

Bouzer, JenN (1945). Grammaire espagnole. Paris. Eugène Belin.

BREsNan, JoAN (1970). On Complementizers: Toward a Syntactic Theory of Complement Types, en Foundalions of Language, 6, pp. 297-32I.

CrOMSKy, NOAM (1957). Syntactic Structures. The Hague. Mouton.

Chomsky, NOAM (1965). Aspects of the Theory of Syntax. Cambridge, Mass. The M. I. T. Press.

CHOMsky, Nonal (1973). Conditions on Transformations, en Anderson, S.; Kiparsky, 1'. (cclis) (1973). A Festscltrift for Morris Halle. New York. Holt, Rinchart and Winston.

Cronsky, NOAN; HLArlu, MORus (1968). The Sound Pattern of English. New York. Harper and Row.

COSTE. J.; REDONDO, A. (1965). Syntaxe de l'espagnol moderne. Paris. SEDES. GIII GAYA, SAATUEI (196r). Cutrso superior de sintaxis española. 8.a ed. Barcelona.

HADLICH, ROGER I. (197I). A Transformational Grammar of Spanish. Englewood Cliffs. Prentice-Hall.

HLARRIS, JANIES (I969). Spanish Phonology. Cambridge, Mass. The M. I. T. Press. InAÑEz, Roberto (1972). Negation im Spanischen. München.

JACKrinom, RAY S. (I $(5)$ ). Specidations on Presentences and Determiners. Bloomington. Indiana liniversity I.inguistics Club.

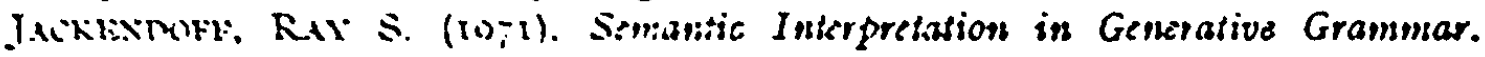

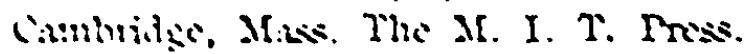

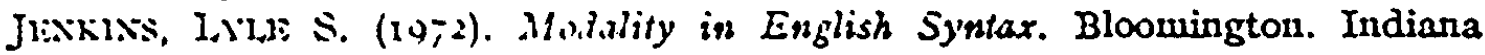
University Linguistics Club.

KI.rin, Pun.1r. W. (1g(is). Modal Albxiliaries in Spanish. Washingfon, D. C. (Iniversity of Washington. Stulies in Linguistics and Language Leaming, vol. IV).

Krinl1, Edward S. (r964). Negalion inz English, en Fodor, Jerry; Katz .Jerrold J. (eds.) (1964). The Structure of Language. Englewood Cliffs. Prentice-Hall.

KÖRNER, KARL-HERMANN (1968). Die Aktionsgemeinschaft finites Verb + Infinitiv im spanischen Formensystem. Vorstudie zu einer Untersuchung der Sprache Pedro Calderon de la Barca. Hamburg (Hamburger Romanistische Studien. B. Tbero-Amerikanische Reihe, Bd. $30=$ Calderoniana, Bd. I).

IENZ, RODOLFO (1935). La oracion y stus partes. Estudios de gramditica generas $y$ castcllana. $3^{\mathrm{a}}$ ed. Madrid. Centro de Estudios Históricos. (Publicacionel de la RFE, 5.) 
KFE, LVII, 19745 EN TORNO A LOS VERBOS PERIFRÁSTICOS DEL ESPAÑOL

MOLINA REDONDO, JOSE ANDRES DE (197I). La construccibn Verbo en forma personal + infinitivon, en Revista Española de Lingütstica, I, pp. 275-298.

OTERO, CARLOS-PEREGRIN (1970). Introducción a la lingǘstica transformacional. México, D. F. Siglo XXI.

Ramsey, Maration Monrosis (1956). A Textbook of Modern Spanish, revised by Ronert IK. Spaulding. New York. Holt, Rinchart and Winston.

REAx ACADEMIA ESPAÑoLA (1973). Esbozo de una mueva gramática de la lengua espanola. Madrid.

RocA Pons, Jost (1958). Estudios sobre perifrasis verbales del español. Madrid. ( $R F E$, Anejo 67).

RUwEr, Nicoras (I968). Introduction da la grammaire générative. Paris. Plon (Recherches en Sciences Humaines, 22).

Schroten, JAN (1972). Concerming the Deep Structures of Spanish Reflexive Sentences. The Hague. Mouton.

SCHROTEN, JAN (1975). The Grammatical Relevance of Conditions on Rules, en A. KRANK (ed.) (1975). Linguistics in the Netherlands, 1972-1973. Assen. Van Gorcuiu.

Sico, Rufari (1962). Manual de gramática española. Madrid. Aguilar.

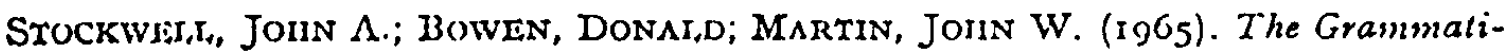
cal Structures of English and Spasish. Chicago (Contrastive Structure Series). 